Démocratie délibérative, démocratie débattante, démocratie participative

\title{
Dans quelles conditions les émotions collectives peuvent-elles bloquer ou aider la délibération démocratique?
}

Pierre Livet

\section{OpenEdition}

\section{Journals}

Édition électronique

URL : http://journals.openedition.org/ress/92

DOI : $10.4000 /$ ress.92

ISSN : $1663-4446$

\section{Éditeur}

Librairie Droz

\section{Édition imprimée}

Date de publication : 1 février 2007

Pagination : 111-128

ISBN : 978-2-600-01114-3

ISSN : 0048-8046

\section{Référence électronique}

Pierre Livet, « Dans quelles conditions les émotions collectives peuvent-elles bloquer ou aider la délibération démocratique? ", Revue européenne des sciences sociales [En ligne], XLV-136 | 2007, mis en ligne le 01 février 2010, consulté le 30 avril 2019. URL : http://journals.openedition.org/ress/92 ; DOI : $10.4000 /$ ress.92 
Pierre LIVET

\section{DANS QUELLES CONDITIONS LES ÉMOTIONS COLLECTIVES PEUVENT-ELLES BLOQUER OU AIDER LA DÉLIBÉRATION DÉMOCRATIQUE?}

La démocratie délibérative se distingue de la démocratie représentative qui donne à tous un droit égal de voter pour tel ou tel représentant, et de la démocratie participative qui permet à tous de participer directement à une décision collective. Elle s'en distingue parce qu'elle demande aux citoyens de participer à l'élaboration des décisions, donc au processus de délibération qui y mène. Toutes ces formes de démocratie sont cependant des essais de réponses au problème politique qui est celui de la décision collective de coopération en incertitude.

Ce problème est le suivant: une action collective pourrait aboutir à un résultat meilleur pour une majorité d'entre nous. Mais d'une part, cette heureuse issue reste incertaine, et d'autre part, y parvenir impose des coûts à la plupart d'entre nous. Enfin, ceux qui s'imposeront ces coûts ne pourront pas empêcher que certains ne les paient pas et jouissent cependant des effets de la coopération. Ces deux derniers inconvénients, eux, sont certains, encore que l'ampleur des comportements de profiteurs reste incertaine. S'il s'agissait pour les citoyens de simplement coordonner leurs actions pour maximiser la satisfaction de leurs intérêts individuels, nous aurions affaire à ce qu'on appelle un problème de coordination. L'incertitude du choix des acteurs entre plusieurs coordinations possibles demeurerait, mais le problème des coûts et des profiteurs n'existerait pas.

On sait que les économistes ont souhaité réduire l'incertitude à des probabilités. Mais les choix politiques se font justement dans des domaines où l'usage des probabilités est assez illusoire. Dans ces domaines, les probabilités que les probabilités estimées soient correctes ne sont pas elles-mêmes précisément estimables. De plus, l'économie - en l'occurrence la théorie des jeux - n'est pas pour l'instant capable de nous dire comment parvenir à des coopérations, au sens que nous venons de définir. Pour la théorie des jeux, la situation que nous venons de décrire correspond à un dilemme des biens publics, et la solution « rationnelle» consiste à ne pas tenter de lancer la coopération.

C'est donc au politique de supporter l'angoisse des tentatives de coopérations. Les régimes politiques ont jusque-là confié le soin de traiter ce problème aux dirigeants ou aux représentants, et c'est le ressort de ce que l'on appelle la démocratie représentative. La démocratie participative, de son côté, ne se montre pas toujours efficace pour surmonter cette difficulté, dès lors que les participants raisonnent en fonction de leur propre intérêt. La démocratie délibérative, en revanche, peut prétendre s'y confronter. En effet, elle se propose bien de partir des intérêts des acteurs, mais aussi de les modifier au cours du processus de délibération de manière à obtenir des coopérations. Son problème est alors double: il s'agit à la fois de motiver les acteurs pour qu'ils coopèrent et entraînent leurs 
semblables, et d'assurer des conditions du processus de délibération qui puissent permettre la sélection de la coopération la plus intéressante.

Nous l'avons dit, ces problèmes de coopération n'ont pas de solution rationnelle coopérative en théorie des jeux, c'est-à-dire dans une théorie du choix en interaction qui ne tient compte que d'une rationalité froide, sans émotion. Notre hypothèse est qu'en revanche ils pourraient en avoir une si l'on tenait compte des processus émotionnels.

Mais inversement, il se révèle que les émotions collectives (que nous restreignons ici à des émotions liées à des coopérations collectives) ne sont pas toutes favorables à la délibération démocratique, et qu'il faut faire quelque tri.

\section{I. ÉMOTIONS DE COMPARAISON, ÉMOTIONS AGIES ET COOPÉRATION}

Nous avons donc d'abord à redécrire le processus de délibération et de décision en tenant compte des émotions. Robert Sugden a développé une tentative intéressante dans ce sens (même si les données expérimentales n'ont pas tranché de manière décisive en faveur de sa théorie), mais notre version sera un peu différente. Le processus fondamental, si on l'étudie au niveau individuel, est le suivant. Nous partons d'une situation présente, et nous envisageons plusieurs possibilités d'action, plusieurs manières de changer le monde. Nous devons aussi tenir compte du fait que le monde environnant change de lui-même. Pour chaque action - il faut aussi compter l'absence d'action - nous devons donc envisager plusieurs résultats, selon que le monde sera plus ou moins favorable au succès de cette action. Nous envisageons donc différentes situations finales, qui résultent de la conjugaison de l'action choisie et de l'état du monde conjugué à cette action. Ces perspectives peuvent susciter deux formes d'émotions. Les unes résultent de la différence entre la situation résultante envisagée et notre situation présente. Si la situation future est meilleure, ce sera une émotion de type positif, et de type négatif dans le cas contraire. Nous pouvons nommer ces émotions «émotions longitudinales », le différentiel étant évalué sur la ligne entre présent et futur. Les autres résultent de la différence entre la situation future envisagée en premier, et une autre situation future, obtenue par une autre action, ou par la même action mais dans un autre état du monde, à laquelle nous la comparons. Nous appellerons ces émotions du second type des «émotions de comparaison» (toutes les émotions impliquent une forme implicite de comparaison, mais ici nous comparons transversalement, en quelque sorte, deux situations futures). Nous nous plaçons par l'imagination dans une situation future - ce futur devient donc notre présent - et nous l'évaluons par rapport à une autre situation contrefactuelle, mais qui aurait pu être la nôtre dans ce présent du futur si nous avions agi autrement ou si l'état du monde avait été différent.

Supposons que nous comparons $\mathrm{Ri}$, un des résultats possibles d'une action $\mathrm{A}$ combinée à tel état du monde Ei avec un des résultats possibles, $\mathrm{Rj}$, d'une action $\mathrm{B}$ combinée à un état du monde $\mathrm{Ej}$, et que le résultat de $\mathrm{A}$ soit moins bon que celui de B. Pour déterminer notre émotion de comparaison, il nous faut encore tenir compte de la confiance que nous avons dans l'obtention du résultat Ri et de celle que nous avons dans l'obtention du résultat $\mathrm{Rj}$. Par exemple, dans une loterie, 
nous avons le choix entre jouer à la loterie ou non. Puis c'est le hasard, donc l'état du monde, qui décide du résultat. L'action A peut donc présenter un résultat favorable Ri (nous gagnons le gros lot) que nous devons comparer d'abord à l'état qui sera le nôtre si nous ne jouons pas à la loterie, état qui sera similaire à notre état présent. Supposons que nous choisissons de ne pas jouer. Imaginons-nous dans la situation future où nous ne l'aurions pas fait. Comparons cette situation avec la situation contrefactuelle où nous aurions joué et gagné le gros lot: nous éprouverons du regret (si nous étions dans l'autre situation, nous éprouverions une grande satisfaction). Mais nous faisons aussi la comparaison entre ne pas avoir joué, et n'avoir rien misé, et la situation où nous aurions misé et aurions perdu notre mise. Nous éprouverons de la satisfaction ou du soulagement de ne pas avoir misé (et dans l'autre situation, nous éprouverions du regret). Or nous avons une confiance excessivement faible dans nos chances de gagner le gros lot, et nous sommes très assurés de ne rien gagner. Nous pouvons donc préférer l'action de ne pas jouer à celle de jouer.

Mais il faut distinguer ici les émotions qui viennent de ce que nous avons agi d'une certaine manière plutôt que d'une autre, et que nous appellerons des émotions agies, et les émotions qui tiennent seulement à ce que le hasard en a décidé ainsi plutôt qu'autrement, et que nous appellerons des émotions subies. Ainsi, nous imaginons la situation dans laquelle le hasard aura décidé que nous n'avons pas gagné le gros lot, et nous savons que nous y éprouverons, par comparaison avec l'autre situation, où nous aurions gagné, de la déception. Inversement nous éprouverions de la réjouissance si nous avions gagné. Notre réjouissance sera d'autant plus grande que nous aurons eu plus de confiance dans l'autre situation, dans le fait de ne pas gagner, et notre déception sera d'autant moins grande que nous aurons eu moins de confiance dans le fait de gagner. Le regret et la satisfaction sont des émotions agies, la déception et la réjouissance sont des émotions subies.

Cependant, ce regret va être pondéré par la confiance très faible que nous avions dans nos chances de gagner le gros lot. Inversement, plaçons-nous dans la situation où nous aurions gagné le gros lot, et comparons-la à la situation où nous n'aurions pas joué. Nous aurons une émotion inverse de celle du regret, une émotion de réjouissance, qui tiendra non seulement à la différence de gain, mais au fait que la situation contrefactuelle, celle où, n'ayant pas joué, nous n'aurions que notre capital actuel, serait certaine. Notre réjouissance sera donc maximale.

Sauf à avoir un tempérament de joueur, qui aime bénéficier d'émotions subies, les émotions dont l'anticipation guide nos choix sont plutôt les émotions agies. Car si, pour reprendre notre exemple, nous n'avons qu'un lot de consolation, nous serons déçu de ne pas avoir le gros lot, mais nous ne nous sentirons pas responsables de ce manque à gagner. C'était le hasard qui décidait. Si en revanche nous avions l'habitude de jouer un numéro, qu'il sorte, mais que ce jour là nous n'ayons pas accompli l'action de jouer, notre regret sera bien plus intense, parce que notre manque à gagner tiendra aussi au choix de notre action.

Cependant, si nous avons alors notre part de responsabilité, puisque nous avons choisi l'action, l'efficace de notre action et son résultat tiennent aussi aux circonstances que nous ne maîtrisons pas. Le problème est alors de savoir comment faire la part de ce qui est subi et de ce qui est agi. Une réponse pourrait être que ce qui donne à chaque agent la garantie qu'il a agi, c'est l'effort qu'il a fait. 


\section{LES ÉMOTIONS DE LA COOPÉRATION}

Mais dans le cas d'une coopération, les circonstances sont en grande partie les actions des autres agents. Et la valeur de l'effort de chacun, qui est sa participation au résultat, tient aussi à ce que les efforts des autres potentialisent cet effort par rapport à ce qu'il pourrait avoir isolément comme résultat, puisqu'il y a un surplus coopératif. La connaissance qu'a l'agent de son effort personnel ne suffit donc pas à assurer la valeur de cet effort, elle lui garantit seulement la possibilité que cet effort participe à la valeur collective. Nous pouvons ici utiliser la notion proposée par Dubois et Prade (Bipolar probabilistic representations, Benferat, Dubois, Kaci, Prade, IRIT, 2004), celle de «possibilité garantie». Les possibilités garanties peuvent se cumuler: si je peux observer l'effort de mes voisins, il m'est raisonnable de penser que cette partie de la coopération est assurée. Si tous ces efforts sont suffisants pour que la coopération réussisse, chacun pourra être satisfait de la coopération à proportion de son propre effort, et pourra se réjouir de l'effort des autres. Mais ici, il n'y a pas d'incertitude. Et les coopérations sociales sont plus exigeantes: les efforts de mes voisins ne suffisent pas, il faut de plus que d'autres acteurs, qui restent pour moi anonymes, participent eux aussi. Si j'en reste à la recherche de possibilités garanties, je ne pourrai obtenir que des informations minimales, et le cumul de ces informations ne me mènera pas bien loin. Le résultat est qu'il restera pour moi irrationnel de me lancer dans la coopération.

Mais il n'est pas très raisonnable d'être toujours aussi prudent. Je pourrais adopter une attitude opposée. Elle consiste à supposer que la coopération reste possible tant que rien ne m'indique que, pour ces acteurs anonymes, elle rencontre des obstacles décisifs. J'utilise alors ce que Dubois et Prade appellent une «mesure de possibilité».

Mais en cas de succès, le coopérateur peut-il s'attribuer tout le résultat coopératif? Les efforts des autres ne comptent-ils pas? Car si nous pouvons considérer nos efforts comme nous assurant une possibilité garantie, nous ne pouvons pas, dans des collectivités, nous assurer des efforts des autres. Dès lors, nous adoptons une attitude prudente à leur égard, puisque nous sommes dans l'incertitude. Pour les résultats possibles des efforts des autres, les résultats qui ne seraient pas en contradiction avec nos attentes, nous ne tablons que sur les résultats minimaux, selon une «mesure (minimale) de possibilité ». Seulement il n'est plus raisonnable de cumuler diverses mesures de possibilité. Puisque nous supposons que tant que nous ne rencontrons pas d'impossibilité, la coopération reste possible, c'est que nous allons au maximum de ce qu'il est possible d'espérer. Si nous avons diverses mesures de possibilité, il est donc raisonnable de ne tabler que sur les mesures minimales.

La différence entre les possibilités garanties et les mesures de possibilité, c'est donc que nous pouvons cumuler les premières, dès que nous avons des observations qui nous les indiquent, alors que nous devons être prudents pour les secondes, et choisir la mesure minimale parmi un éventail de mesures possibles.

Mais il y alors de fortes chances pour que, si nous faisons la somme des mesures minimales de possibilité des efforts des anonymes, et des données de possibilité garantie de notre propre effort et de ceux de nos voisins, cela ne suffise pas à assurer la réussite de la coopération. Cela n'exclut cependant pas que la somme des efforts effectifs de chacun, donc la somme des possibilités que chacun 
peut garantir pour lui-même mais qu'il ne peut pas garantir pour les autres, pourrait assurer le résultat coopératif. Mais le problème est que nous n'avons pas accès aux efforts des anonymes (et pas totalement aux intentions d'efforts de nos voisins). Par ailleurs, nous ne savons pas comment les efforts que nous observons vont pouvoir se combiner avec ceux que nous n'observons pas. Cette combinaison ne sera pas forcément réussie, les efforts des uns peuvent s'entre empêcher avec les efforts des autres, etc. Et chacun sait que les autres sont dans une situation similaire concernant ce manque d'information et cette incertitude sur la bonne marche de la coopération. Sachant cela, chacun peut être tenté de ne pas activer son effort autant qu'il lui serait possible, et qu'il serait nécessaire pour assurer la réussite de la coopération.

Cependant celui qui coopère fait comme s'il pouvait faire confiance à ceux qu'il ne peut observer pour assurer un effort qui est au-delà de l'effort minimal (dont nous avons supposé qu'il ne permet pas la réussite de la coopération). Il va au-delà des possibilités garanties dont il dispose, tout en restant en deçà d'une généralisation de ces possibilités garanties, et il va aussi au-delà d'une estimation du minimum des mesures de possibilité qui soit elle-même révisée à la baisse en raison des incertitudes que chacun a sur les efforts des autres qui sont pour lui anonymes.

Supposons maintenant que la coopération réussisse. Nous voyons que notre environnement social permet maintenant certaines actions qui étaient autrefois impossibles. A qui pouvons-nous assigner le mérite de cette réussite? Nous ne pouvons pas baser ce mérite sur ce que nous avons observé des efforts de chacun, puisque nos observations se sont bornées à notre voisinage. Nous ne pouvons pas non plus nous dire que puisque la coopération a réussi, c'est que les efforts de chacun étaient suffisants, et que chacun de nous individuellement peut s'en attribuer le mérite. Il se peut que certains aient agi comme si les autres faisaient des efforts importants, donc en extrapolant les possibilités garanties qu'ils ont observées, alors que d'autres auront agi de manière à tenter de réussir même si les actions des autres n'atteignaient que le minimum de la mesure de possibilité. Les premiers auront donc fait un effort minimal, parce qu'ils supposaient que les autres feraient un effort important, et les seconds auront fait un effort maximal, parce qu'ils tenaient compte du fait que les autres pourraient n'avoir agi que minimalement. Et nous ne pouvons pas savoir qui, parmi ceux qui ont agi hors de portée de nos observations, en a fait trop par rapport à ce qui était en fait nécessaire, ou pas assez. Davantage: dans une activité de coopération, si nous en faisons beaucoup plus que les autres, parce que nous n'avons pas confiance dans l'effort des autres, nous les incitons à en faire moins, puisque nous leur montrons que nous n'avons pas confiance dans leur capacité à coopérer de manière efficace avec nous. En agissant ainsi, nous nous donnons des justifications pour nous attribuer un mérite individuel, mais nous diminuons l'effet que nous avons sur la collectivité en tant que telle, et donc notre mérite dans la coopération. Celui qui a le plus de mérite, ce n'est donc pas celui qui en fait le plus individuellement - et ce n'est évidemment pas non plus celui qui en fait le moins. C'est celui qui a la juste confiance dans les autres et aussi la juste inquiétude concernant la réussite de la coopération. On ne peut donc déterminer ce mérite qu'après coup, une fois la coopération réussie. Mais le mérite tient alors à la différence entre le succès de la coopération et les attentes raisonnables que l'on pouvait avoir avant son succès. Si j'ai sous-estimé 
ces attentes, et que j'ai cependant tenté de coopérer, j'ai dû faire un effort excessif et qui montrait mon peu de confiance dans les autres. Si en revanche j'ai surestimé la participation des autres et les chances de réussite de la coopération, j'ai fait trop peu d'effort et j'ai donc peu de mérite. Mais le fait que je me trouve avoir fait exactement la juste estimation ne peut pas me donner l'émotion «agie» d'une satisfaction, puisqu'en fait cette estimation s'est révélée juste non pas en raison de ma perspicacité, mais en raison d'effets collectifs sur lesquels je n'ai pas de prise et qui ne peuvent donc susciter en moi qu'une émotion «subie». Or cette émotion ne peut me permettre de m'attribuer un mérite. Pourtant, collectivement, et par notre action collective, nous pouvons prétendre à une émotion agie, et nous attribuer le mérite de la coopération, puisqu'elle a tenu à ce que nous avons tous pris le risque et fait l'effort de coopérer.

Ainsi le mérite individuel dans une coopération ne tient pas qu'à l'effort individuel. Cet effort est en effet le signe du degré de confiance que j'ai dans les efforts des autres. Mon mérite suit une courbe en U inversé: quand j'ai une grande confiance dans l'effort des autres, je fais peu d'effort, et j'ai peu de mérite. Quand j'ai une très faible confiance, je fais beaucoup d'efforts, mais, montrant mon peu de confiance, j'incite les autres à en faire moins, s'ils me font confiance, ou à se décourager, puisqu'ils en déduisent que la coopération a très peu de chances de réussir. Comme mon mérite dépend de ce degré de confiance des autres, il dépend des interactions propres à la collectivité. Mon mérite n'est donc pas individuel, il est collectif.

Mais même une fois la coopération réussie, nous ne pouvons toujours pas savoir quelle était la différence entre le succès de la coopération et les attentes effectives des individus, puisque nous ne connaissons pas toutes ces attentes. Nous devons dons supposer qu'une différence raisonnable existait, mais cette différence raisonnable tient aussi aux interactions collectives, puisqu'elle varie en fonction des variations de confiance. Ainsi non seulement la source du mérite reste collective, mais l'estimation du mérite aussi.

Ainsi, celui qui fait un effort raisonnable de coopération est celui qui s'appuie sur ce qu'il observe comme efforts autour de lui et qui fait confiance aux autres pour espérer que de tels efforts vont se poursuivre, peut-être de manière moins intense, sur tout l'ensemble de la collectivité, alors même que s'il en restait à une attitude prudente, il devrait ne se fier, pour les individus qu'il ne peut observer, à un effort minimal. Mais cette différence entre la généralisation des possibilités garanties et la généralisation des mesures de possibilité minimales ne peut déterminer le mérite d'un individu qui coopère que si les autres ont bien répondu à sa confiance en faisant la même confiance à leurs coopérateurs anonymes. Et pour qu'ils y répondent correctement, il faut qu'il se trouve avoir choisi le bon niveau de confiance. Mais ce bon niveau de confiance dépend des interactions collectives, et il n'est au pouvoir d'aucun des individus de le fixer.

Nous allons donc attribuer le mérite de la coopération, le mérite estimé par ce surplus des efforts faits par confiance sur les efforts faits par défiance, à un acteur collectif, puisque nous ne pouvons pas savoir quel est le bon niveau de confiance sinon en retour des interactions collectives, et que nous, agents individuels, participons à cet acteur collectif, de manière collective, parce qu'indissociable. Ce qu'obtient cet acteur collectif ne doit pas pour autant être considéré par les acteurs individuels comme subi. En effet, cet acteur ne fait rien d'autre que coordonner des actions individuelles qui se potentialisent les unes les autres. 
Inversement, si l'issue de la coopération était certaine (donc si elle se réduisait à une simple coordination), l'acteur collectif pourrait se réduire à la somme des efforts de chacun. En effet, la différence entre la somme des possibilités d'effort garanties de manière observable pour chaque individu et la somme des efforts réduite à une mesure de possibilité minimum, cette différence se réduirait à zéro. Dans ce cas, le surplus collectif se réduit à zéro, et l'acteur collectif se réduit aussi par là même à zéro.

Or, quand au contraire l'acteur collectif résulte de cet intervalle d'incertitude acceptée, nous pouvons aussi disposer des émotions de base et des émotions de comparaison qui sont à la mesure du pouvoir de cet acteur collectif, et non plus des émotions qui sont seulement à la mesure de notre effort individuel.

Il reste maintenant à montrer que la décision de coopérer, une fois tenu compte des émotions positives liées à la participation à cet acteur collectif, est bien rationnelle.

Notons d'abord que la situation proposée par le dilemme du prisonnier ne reflète pas exactement la situation de coopération ici envisagée. Car dans le DP, si un agent a tenté de coopérer, l'autre agent qui fait défection obtient le gain maximal (celui de l'exploitation), alors que le coopérant a perdu le coût de son effort. Mais c'est supposer qu'il suffit qu'un seul agent coopère pour que le bénéfice de la coopération soit obtenu, et que cependant cet agent coopérant n’en bénéficie pas! Ce que nous envisageons comme situation de coopération, ce sont des situations collectives plus courantes, où le gain de l'exploitation est maximal non pas quand les coopérants n'obtiennent pas le gain de la coopération, mais quand une majorité coopère, et que le gain de la coopération est bien obtenu. En revanche, quand la coopération ne marche pas - parce que l'exploitation s'est généralisée le seul gain des exploiteurs est d'avoir économisé l'effort de coopérer. Dans toutes ces situations, il est donc possible de coopérer, d'avoir le gain de la coopération, et qu'un autre ait de plus le gain de l'exploitation. Quand les exploiteurs sont trop nombreux, ils n'obtiennent pas le gain de l'exploitation, ils se bornent à économiser l'effort qu'a au contraire fait le coopérant, qui est donc dans la situation la pire. Mais la matrice de cette situation peut donner aussi un équilibre de Nash de défection ou d'exploitation mutuelle, puisque quand suffisamment de coopérants coopèrent, il est plus avantageux de les exploiter, et que quand il y a trop d'exploiteurs, le coopérant est celui qui perd le plus.

Que se passe-t-il en cas d'échec de la coopération? Le raisonnement usuel nous dit que si nous avons agi coopérativement, nous avons enduré un effort coûteux, alors que si nous n'avons rien fait, nous nous sommes au moins épargné cet effort.

Les choses sont différentes selon que nous considérons les émotions purement individuelles, et celles que nous éprouvons en tant que participant à l'action collective de coopération. Or, il nous faut maintenant intégrer ces émotions dans notre évaluation. Celui qui coopère ne peut ressentir qu'une émotion subie (sauf si le pari qu'il avait fait sur la coopération se révèle après coup reposer sur une estimation ridiculement optimiste), puisqu'il a fait l'effort nécessaire, que d'autres l'ont fait aussi, mais que les circonstances ont été défavorables (il peut aussi ressentir du ressentiment envers ceux qui n'ont pas coopéré, nous y reviendrons).

Celui qui a fait défection a choisi de ne pas participer à l'action collective. Considérons alors l'émotion de comparaison qu'il éprouve en comparant sa situa- 
tion à celle qu'il aurait eue si la coopération avait réussi, et qu'il ait pu profiter du bénéfice de l'exploitation. Est-ce une émotion subie ou une émotion agie? Si la décision de ne pas coopérer a été prise avec l'intention de profiter de l'exploitation, il s'agit d'une décision agie et responsable, prise contre une majorité d'autres acteurs sociaux, et l'émotion est donc un regret, une émotion agie. Ce ne peut être une émotion subie, une déception, que si la coopération apparaissait très incertaine, et que notre homme a suivi une majorité. Mais alors il ne s'attendait pas à pouvoir bénéficier de l'exploitation, il a simplement été prudent.

Inversement, quand la coopération réussit, celui qui a coopéré bénéficie de l'émotion de l'action collective, une émotion qui est agie, comme nous l'avons vu. Celui qui a refusé de coopérer s'est forcément privé de cette émotion collective positive. Peutil éprouver pleinement l'émotion agie qui tient à la réussite de son projet d'exploitation? Non, parce que cette réussite ne tient pas à lui ou au collectif dont il ferait partie - puisqu'il a refusé d'en faire partie - mais à l'efficacité de l'effort des autres. Son émotion n'est donc que partiellement une émotion agie, et partiellement une émotion subie, due à des circonstances extérieures. Et nous avons dit que les émotions subies étaient moins décisives pour nos choix d'action que les émotions agies.

A partir de ces données, nous pouvons maintenant évaluer les émotions (de base et de comparaison) dans toutes les situations possibles. Notons pour la suite $\mathrm{C}+$ la réussite collective de la coopération, $\mathrm{C}$ - son échec, $\mathrm{c}+$ une coopération individuelle, et c- la défection individuelle. Notre agent doit donc évaluer les différentes combinaisons suivantes:

1) en cas de réussite de la coopération, s'il a coopéré $(\mathrm{C}+, \mathrm{c}+)$, il compare cette situation aux situations où

a) la coopération n'aurait pas réussi et où il aurait coopéré $(\mathrm{C}+, \mathrm{c}+) /(\mathrm{C}-, \mathrm{C}+)$

b) la coopération aurait réussi et où il aurait refusé de coopérer $(\mathrm{C}+, \mathrm{c}+) /$ $(\mathrm{C}+, \mathrm{c}-)$

c) la coopération n'aurait pas réussi et où il aurait refusé de coopérer $(\mathrm{C}+, \mathrm{c}+) /$ (C-, c-).

2) en cas d'échec de la coopération, s'il a coopéré (C-, c+), il compare cette situation aux situations où

a) la coopération aurait réussi et il aurait coopéré $(\mathrm{C}-, \mathrm{c}+) /(\mathrm{C}+, \mathrm{c}+)$

b) la coopération aurait réussi et il aurait refusé de coopérer $(\mathrm{C}-, \mathrm{C}+) /(\mathrm{C}+, \mathrm{c}-)$

c) la coopération n'aurait pas réussi et il n'aurait pas coopéré $(\mathrm{C}-, \mathrm{c}+) /(\mathrm{C}-, \mathrm{c}-)$

3) en cas de réussite de la coopération, s’il n'a pas coopéré $(\mathrm{C}+, \mathrm{c}-)$, il compare cette situation aux situations où

a) la coopération aurait réussi, et il aurait coopéré $(\mathrm{C}+, \mathrm{c}-) /(\mathrm{C}+, \mathrm{c}+)$

b) la coopération n'aurait pas réussi et il aurait coopéré $(\mathrm{C}+, \mathrm{c}-) /(\mathrm{C}-, \mathrm{c}+)$

c) la coopération n'aurait pas réussi et il n'aurait pas coopéré $(\mathrm{C}+, \mathrm{c}-) /(\mathrm{C}-, \mathrm{c}-)$

4) en cas d'échec de la coopération, s'il n'a pas coopéré (C-, c+), il compare cette situation aux situations où

a) la coopération aurait réussi et il aurait coopéré $(\mathrm{C}-, \mathrm{c}-) /(\mathrm{C}+, \mathrm{c}+)$

b) la coopération aurait échoué et il aurait coopéré $(\mathrm{C}-, \mathrm{c}-) /(\mathrm{C}-, \mathrm{c}+)$

c) la coopération aurait réussi et il n'aurait pas coopéré $(\mathrm{C}-, \mathrm{c}-) /(\mathrm{C}+, \mathrm{c}-)$. 
Dans ce qui suit, nous allons nous placer dans une situation socialement des plus plausibles: celui qui exploite la coopération des autres fait un profit supérieur évidemment à celui qui coopère, mais son profit ne consiste que dans ce qu'il épargne le coût de la coopération, et ce coût n'est pas dirimant. Il en est en effet ainsi des plus nombreux exploiteurs dans nos sociétés: ceux qui fraudent le fisc et qui échappent à des pénalités - alors que les autres paient leurs impôts. Par ailleurs, comment devons nous tenir compte de ce moindre poids des émotions subies par rapport aux émotions agies dans nos décisions? Ici, pour simplifier, nous supposerons simplement que l'introduction d'une émotion subie diminue la contribution de cette émotion à l'évaluation comparative d'une situation. C'est comme si cela diminuait l'intensité de cette émotion, alors qu'en fait cela diminue essentiellement son poids pour notre choix d'action.

Par rapport à $1 \mathrm{a},(\mathrm{C}+, \mathrm{c}+) /(\mathrm{C}-, \mathrm{c}+)$, le coopérant se réjouit d'avoir une émotion positive et agie, puisque si la coopération tient aussi aux autres, il a bien fait l'effort qui lui permet de participer à cette réjouissance collective: $(+++)$

Par rapport à $1 \mathrm{~b},(\mathrm{C}+, \mathrm{c}+) /(\mathrm{C}+$; $\mathrm{c}-)$, le coopérant compare son succès au succès de l'exploiteur, qui serait partiellement subi, puisque si tout le monde avait agi comme lui, il n'y aurait pas eu de coopération, et qu'il a donc fallu que d'autres agissent en sens inverse. Le coopérant enregistre en revanche le gain de la coopération, selon une réjouissance non affaiblie. L'avantage est à l'exploiteur, mais, en supposant que son avantage ne consiste que dans le fait de ne pas subir les coûts de la coopération, il est encore affaibli parce qu'il est subi au lieu d'être agi, ce qui donne pour le coopérant un regret faible: (-). Il est même possible que le regret soit nul, puisque cet avantage de l'exploitation exigerait de renoncer au partage collectif d'une émotion positive: (0).

Par rapport à $1 \mathrm{c},(\mathrm{C}+, \mathrm{c}+) /(\mathrm{C}-, \mathrm{c}-)$, il se réjouit aussi d'avoir une émotion agie positive, au lieu d'une émotion négative qui serait agie (puisque le refus de coopérer participe à l'échec de la coopération) et du simple soulagement de ne pas avoir fait l'effort de coopérer: (++).

Pour ne pas entrer dans des raisonnements compliqués sur les différentes manières d'agréger ces différentes émotions, nous les additionnerons. Ce n'est pas ainsi que les choses se passent pour les affects, mais ce qui nous intéresse ici est le bilan global, et nous pouvons arriver par une méthode contestable à un résultat qui n'est pas très différent de ce que serait la combinaison effective des émotions. Le total de nos émotions donnera donc pour la situation $1:(+++++;-$ ou 0$)$

Par rapport à $2 \mathrm{a},(\mathrm{C}-, \mathrm{c}+) /(\mathrm{C}+, \mathrm{c}+)$, le coopérant regrette que les circonstances aient été défavorables, mais le mauvais résultat tient seulement aux circonstances, donc son émotion est affaiblie (-).

Par rapport à $2 \mathrm{~b},(\mathrm{C}-, \mathrm{c}+) /(\mathrm{C}+; \mathrm{c}-)$ : dans la situation à laquelle il se compare, la coopération réussie de la situation contrefactuelle tiendrait essentiellement aux circonstances (malgré le refus de coopérer, les circonstances auraient produit la coopération, et ce seraient donc des circonstances exceptionnelles). Donc l'émotion de regret est très affaiblie (-).

$2 c,(\mathrm{C}-, \mathrm{c}+) /(\mathrm{C}-, \mathrm{c}-)$, le coopérant a-t-il une émotion de regret d'avoir fait l'effort (finalement vain) de coopérer? Il a bien fait cet effort, mais le fait qu'il soit vain ne lui est pas dû. La qualité négative de l'effort ne tient pas à cet effort, mais 
aux circonstances, donc ce regret est lui aussi lié à une émotion subie, et est affaibli. (-)

Le total de la situation 2 donne: (--)

Par rapport à $3 \mathrm{a},(\mathrm{C}+, \mathrm{c}-) /(\mathrm{C}+, \mathrm{c}+)$, l'exploiteur se réjouit d'avoir le bonus de l'exploitation, mais si son refus de coopérer est agi, la réussite de la coopération ne tient nullement à lui, si bien que la qualité positive que se trouve avoir pris son refus est partiellement subie. Sa réjouissance est donc affaiblie. Si son avantage est déjà réduit à échapper au coût de la coopération, il est encore affaibli $(+)$.

Par rapport à $3 b,(\mathrm{C}+, \mathrm{c}-) /(\mathrm{C}-, \mathrm{c}+)$, il se réjouit de n'avoir pas fait l'effort de coopérer (et cela est agi, puisque comparé à un effort de coopération). En revanche, la réjouissance qui porte sur le fait que la coopération a réussi est toujours une émotion subie, donc affaiblie. Là encore, l'émotion positive n'est forte qu'en partie $(++)$.

Par rapport à $3 \mathrm{c},(\mathrm{C}+, \mathrm{c}-) /(\mathrm{C}-, \mathrm{c}-)$, il ne peut se réjouir que faiblement de la réussite de la coopération, puisque pour lui cette réussite est subie $(+)$.

Le total de la situation 3 donne $(++++)$.

Par rapport à $4 \mathrm{a},(\mathrm{C}-, \mathrm{c}-) /(\mathrm{C}+, \mathrm{c}+)$, notre exploiteur a un regret qui est agi, puisqu'il est responsable de l'échec de la coopération pour avoir refusé de coopérer, et qu'il partage avec les autres cette responsabilité (--).

Par rapport à $4 \mathrm{~b},(\mathrm{C}-, \mathrm{c}-) /(\mathrm{C}-, \mathrm{c}+)$, l'exploiteur se réjouit de ne pas avoir fait l'effort de coopérer; c'est une émotion agie, mais dont la base est faible (+).

Par rapport à $4 \mathrm{c},(\mathrm{C}-, \mathrm{c}-) /(\mathrm{C}+; \mathrm{c}-), \mathrm{il}$ a un regret qui est agi, puisqu'il partage avec les autres la responsabilité de l'échec de la coopération (--), et il faut y ajouter le regret subi que l'exploitation n'ait pas été possible (-). Le total pour 4c est (---).

Le total de la situation 4 est donc de: $(-----,+)$.

\begin{tabular}{|c|c|c|c|c|c|}
\hline $\begin{array}{l}\text { Comparaison } \\
\text { de...I vers }->\end{array}$ & $\begin{array}{l}\mathrm{C}+; \mathrm{c}+ \\
\text { coopération }\end{array}$ & $\begin{array}{l}\mathrm{C}-; \mathrm{c}+ \\
\text { échec }\end{array}$ & $\begin{array}{l}\mathrm{C}+; \mathrm{c}- \\
\text { exploitation }\end{array}$ & $\begin{array}{l}\text { C-; c- } \\
\text { défection }\end{array}$ & Total \\
\hline $\mathrm{C}+; \mathrm{c}+(\mathrm{Coop})$ & & $1 \mathrm{a}(+++)$ & $1 \mathrm{~b}(-)$ ou 0 & $1 \mathrm{c}(++)$ &,+++++- ou 0 \\
\hline C-; c+ (Echec) & $2 \mathrm{a}(-)$ & & $2 b(-)$ & $2 \mathrm{c}(-)$ & --- \\
\hline C+; c- (Exploit) & $3 a(+)$ & $3 \mathrm{~b}(++)$ & & $3 \mathrm{c}(+)$ & ++++ \\
\hline C-; c- (Defec) & $4 \mathrm{a}(--)$ & $4 \mathrm{~b}(+)$ & $4 c(---)$ & &,-----+ \\
\hline
\end{tabular}

Si nous pouvions faire des additions, le coopérant l'emporterait de peu sur l'exploiteur ( 5 plus et 4 ou 3 moins, contre 5 plus et 5 moins). Et si l'on considère les émotions négatives et positives maximales, le coopérant bénéficie de l'émotion positive maximale, et l'exploitant de l'émotion négative maximale. Il semble donc que le bilan soit en faveur du coopérant, dès que l'avantage de l'exploitation se réduit à l'absence de participation au coût de la coopération.

Cela tient à trois facteurs: 1) l'exploiteur ne peut pas participer activement au succès de la coopération, alors qu'il participe activement à l'échec de la coopération. Il se prive donc d'une émotion positive active forte et collective, et prend le fort risque d'une émotion négative active assez forte et partagée, quand la coopé- 
ration échoue, faisant échouer par là même l'exploitation. 2) Quand l'exploiteur obtient son gain maximal (si la coopération réussit), il en doit l'essentiel aux circonstances et non à son action (c'est donc pour une bonne part une émotion subie). 3) Le coopérant, en cas d'échec, n'a pas à imputer cet échec à son action (il a fait tout ce qu'il fallait faire pour que la coopération réussisse) mais aux circonstances, ce qui affaiblit son émotion négative (cela, si l'on suppose qu'il n'a pas tenté une coopération alors que la majorité des autres lui était hostile!).

\section{LES DIFFÉRENTS RÔLES DES ÉMOTIONS COLLECTIVES PAR RAPPORT À LA DÉLIBÉRATION DÉMOCRATIQUE}

Nous avons montré que les décisions collectives de coopération en incertitude, en quoi consistent les décisions politiques, sont justifiées par un bilan qui s'appuie sur des émotions de comparaison. Ce bilan va de pair avec la constitution d'un intervalle entre la garantie de l'effort de chacun et le minimum d'effort supposé pour les autres. Le comblement de cet intervalle donne lieu à la constitution d'un acteur collectif, nos émotions nous permettant alors une participation aux émotions collectives assignées à la globalité de cet acteur. Autrement dit, un tel dispositif crée une différence entre l'acteur public collectif et les acteurs purement privés.

Une des raisons pour lesquelles nous adoptons des décisions politiques qui supposent des coopérations en situation d'incertitude (qualification qui est maintenant redondante avec la notion même de décision politique), c'est donc qu'elles nous proposent le bénéfice des émotions de participation à cet acteur collectif. Ce n'est pas que nous soyons ici à la recherche d'un maximum d'émotions agréables et d'un minimum d'émotions désagréables. C'est simplement que lorsque nous devons choisir entre deux actions, dont l'une est coopérative et l'autre non, les émotions que nous ressentons en anticipant les différentes situations possibles et en faisant des comparaisons nous rendent sensibles aux différentiels entre les situations, et que ces orientations différentielles, en s'agrégeant, guident nos décisions finales. Et de plus, ces émotions collectives de participation à une coopération sont des émotions qui ne nous sont accessibles que si nous prenons des risques collectifs plus élevés qu'une prudence purement individuelle ne l'imposerait.

Mais d'autres émotions interviennent dans les délibérations qui conduisent à des décisions politiques. Tout d'abord, les délibérations sont déjà des coopérations en incertitude. Elles provoquent donc les émotions collectives que nous venons de voir, lorsque la délibération paraît avoir atteint ses objectifs. Mais les délibérations mettent en jeu bien d'autres émotions que celles de coopération: des émotions liées aux tensions, blocages ou avancées de la négociation, des émotions d'appartenance à un groupe, de réaction contre un autre groupe, des émotions de ressentiment contre ces autres groupes, voire contre ceux qui ont engagé la délibération dans tel ou tel sens.

Nous entendons ici «délibération» au sens le plus large possible. Dès que des membres d'une population réunissent leurs opinions et discutent soit pour décider d'une action collective, soit pour donner des arguments et faire valoir leur point de vue pour un problème qui concerne une telle décision collective, il y a 
délibération. Cette délibération est démocratique si elle n'exclut pas des personnes concernées par la décision, si elle permet d'entendre les différentes positions et arguments, si elle ne mène pas à une décision contrainte par la violence, et si une majorité des personnes concernées peut manifester son acceptation - et les autres leur refus - de la décision proposée. La délibération n'est donc pas restreinte au gouvernement, ou à des institutions parlementaires. Il n'est pas nécessaire non plus que les personnes soient en présence les unes des autres, mais seulement que des moyens de transmission de l'information permettent à chacun de se faire entendre et d'accéder aux opinions des autres et aux informations que les autres avancent. Les réactions de l'opinion publique (manifestations, envois de messages, etc.) à une décision gouvernementale font ainsi partie de la délibération entendue en ce sens large. Dès lors, ne pas tenir compte des émotions dans la délibération politique serait suicidaire, puisque ces émotions peuvent intervenir dans la délibération et modifier considérablement l'impact des décisions prises.

Il peut être utile de relier les émotions à leur origine temporelle. Les émotions peuvent se diviser entre émotions reliées à des situations passées, à des situations futures, à des situations présentes. Il est de plus très utile de tenir compte des émotions qui se sont manifestées par le passé (et qui pouvaient elles-mêmes porter sur le présent, sur le passé, ou sur l'avenir). En effet, les émotions collectives qui se sont manifestées précédemment sont indispensables pour nous révéler les valeurs effectives des agents, sans en rester aux valeurs qu'ils affichent, puisque ces émotions guident leurs choix, et que les valeurs effectives ont aussi cette fonction (il est possible que certaines valeurs ne suscitent pas en nous d'émotions, mais elles auront alors du mal à guider nos choix ${ }^{1}$ ).

Une délibération politique met en jeu plusieurs acteurs collectifs (plusieurs groupes qui représentent des intérêts et des valeurs). Mais ces groupes peuvent déjà se sentir membres d'un acteur collectif plus large, par exemple une région ou une nation. Les émotions propres à ces différents acteurs collectifs et qui se sont déjà manifestées avant la délibération sont très utiles pour que chacun des groupes puisse savoir quelles sont les valeurs auxquelles les autres sont attachés. Pendant la délibération, ces émotions de participation à l'acteur collectif vont permettre des partages émotionnels et une motivation collective plus forte pour aboutir à une décision. Tenter de les éliminer pour permettre une démarche supposée plus rationnelle, c'est prendre le risque de faire échouer la délibération, ou que son résultat donne lieu à plus de ressentiment qu'au départ.

Si nous nous bornons à étudier les émotions du passé, du présent et de l'avenir dans l'interaction entre dirigeants et citoyens, il est évident qu'une décision gouvernementale ou parlementaire qui ne tient pas compte des émotions manifestées par sa population par le passé coupe la politique de la nation, et fait obstacle au jeu de la délibération au sens large.

De manière symétrique, un gouvernement qui prend une décision en ne se souciant que de ses conséquences utilitaires, sans se soucier de la résonance émotionnelle des actions ainsi engagées, s'expose à des retours de flamme violents et ne

1 Nous ne pouvons développer plus longuement ce point ici, et renvoyons à Emotions et Rationalité morale, Paris, PUF, 2002. 
comprend tout simplement pas quelle est sa tâche dans la perspective élargie d'une démocratie délibérative. Il faut en particulier bien se rendre compte que le fait qu'une décision soit prise par une instance qui décide de manière isolée et sans interaction avec les personnes concernées par cette décision suscite évidemment une émotion politique fondamentale, celle du ressentiment. Cela ne veut pas dire que le politique ne doit jamais risquer de susciter du ressentiment - on ne pourrait jamais agir s'il en était ainsi -, mais que le politique doit tenir compte du ressentiment qu'il a suscité, et tenter de le désamorcer par d'autres mesures, ou par une plus grande participation à la délibération.

Quelles sont maintenant les émotions au présent de la délibération? Nous avons déjà indiqué les émotions de coopération et les émotions propres à la dynamique de la négociation. Nous avons déjà analysé les premières. Faute de place, nous ne pourrons ici analyser les secondes, les dynamiques émotionnelles exigeant des études qui sont compliquées à exposer. Nous allons donc nous concentrer sur une troisième sorte d'émotions induites dans la délibération au sens large, et que nous pouvons appeler de manière générique les émotions altéro-réactives. Nous avons déjà cité le ressentiment, mais nous pouvons aussi mentionner la rivalité, ou encore la rancœur que nous pouvons ressentir à l'idée qu'un autre groupe puisse ressentir une joie maligne à voir notre situation dégradée par rapport à la sienne. Sous cette dernière forme il s'agit d'une réaction émotionnelle d'un groupe à l'idée que cet autre groupe éprouverait en propre telles autres émotions, émotions qu'il éprouverait lui-même à l'idée que la dégradation de notre situation provoque dans notre groupe des émotions négatives - y compris cette émotion de rancœur.

Nous avons là des émotions qui peuvent amener d'abord l'échec d'une coopération, quand un sous-groupe se met à soupçonner un deuxième sous-groupe de n'en faire pas assez et de se réjouir de bénéficier des efforts du premier sans avoir apporté sa propre contribution. Mais elles peuvent aller jusqu'à susciter une sorte d'anti-coopération: les membres du sous-groupe vont faire certains efforts, qui vont parfois contre leurs intérêts, pour briser la coopération, et ils vont ressentir dans cette affaire des émotions collectives, et constituer une acteur collectif anticoopératif. Les révoltes de groupes sociaux qui n'espèrent pas d'amélioration de leur sort (jacqueries, révoltes des banlieues) sont de ce type.

Cette classe d'émotions collectives peut rendre la démocratie impossible à réformer, dès que ces émotions reposent sur des ressentiments, et en provoquent chez ceux dont les coopérations sont ainsi mises en danger. Si nous interprétons les ressentiments dans les termes des coopérations, ils tiennent à ce que des groupes s'aperçoivent après coup qu'ils ont coopéré avec des acteurs sociaux qui les ont exploités, donc qui ont transformé ce qui aurait pu être une situation d'action en incertitude en une situation dont l'incertitude est subie. Tant que cette exploitation n'est pas reconnue et que ses conditions ne sont pas modifiées, le groupe de ceux qui découvrent avoir été exploités et celui des exploiteurs ne peuvent pas réellement participer conjointement à un processus de délibération démocratique. Les négociations qui peuvent avoir lieu resteront alors des négociations entre les représentants des uns et des autres. Cette démocratie représentative au sens large (au sens où toute délibération entre des représentants sélectionnés selon un processus institué et en accord avec une constitution est une pièce 
fondamentale de la démocratie représentative) est donc nécessaire, du point de vue même de la démocratie délibérative au sens large, pour faire redémarrer des relations alors même que la délibération démocratique est bloquée. Mais on comprend aussi pourquoi la démocratie représentative apparaît, du point de vue de ceux qui éprouvent ce ressentiment, comme une manière de repousser le traitement du problème.

La constitution d'un acteur anti-coopératif part en fait d'émotions négatives qui sont d'abord subies - puisqu'elles tiennent à une dégradation de la situation de notre propre groupe. Si nous pouvons attribuer au moins partiellement cette dégradation à un autre groupe, notre opposition à ce groupe et à la réussite des coopérations qu'il organise sera le moyen de passer d'émotions subies à des émotions agies. Nous coopérons alors entre nous, mais seulement pour nous opposer à la réussite des coopérations de l'autre groupe. Nous constituons un acteur collectif, mais anti-coopératif. Nous voulons punir autrui, donc nous allons au-delà de la défection mutuelle. Nous acceptons de nous priver pour punir (Fehr a montré que ce coût que s'imposent les punisseurs face aux exploiteurs est décisif pour permettre l'instauration de coopération). Mais nous ne pouvons faire cela de manière politique que si notre acteur coopératif met en place de nouvelles coopérations. Car alors ces efforts pour punir font partie de la révélation de la valeur de coopération positive. Les seules coopérations nécessaires aux sabotages de l'acteur anti-coopératif ne permettent pas de mettre en place une démocratie, qui exige que les efforts convergent pour assurer la réussite d'une coopération, et non pas seulement que le sabotage d'une coopération rende nécessaires certains efforts. L'acteur anti-coopératif ne peut exister sans la coopération qu'il tente de saboter, il ne construit pas un nouvel acteur collectif par synergie à partir des acteurs collectifs précédents.

\section{LE RESSENTIMENT}

Cependant, le ressentiment est une émotion essentielle pour toute démocratie, du moins s'il s'agit du ressentiment qui vient de la découverte que ce que l'on tenait pour une coopération entre groupes donnait en fait essentiellement lieu à une exploitation par un des groupes. La coopération a réussi, mais ceux qui en supportent les efforts s'aperçoivent que d'autres, sans faire autant d'efforts, en retirent bien davantage. Ce ressentiment sera évidemment attisé par le soupçon que ces profiteurs ont paru coopérer alors qu'ils avaient dès le départ l'intention de prendre moins de risques et d'en tirer plus de résultats. Mais il peut même porter sur ceux qui ont effectivement pris des risques supérieurs à ceux pris par les coopérateurs, en pariant sur la réussite de la coopération, mais qui retirent de cette prise de risque des avantages qui leur sont systématiquement réservés. Chacun des coopérateurs admet des disparités dans les avantages retirés, mais ces avantages «normaux» de la coopération respectent l'incertitude de l'attribution des bénéfices aux différents coopérants. Ils ne peuvent donc pas être systématiques. Cela laisse soupçonner que ces profiteurs ont bien pris des risques, mais qu'ils en retirent des avantages qui n'obéissent pas à l'incertitude du risque propre à la coopération. On pourrait dire qu'ils ont transformé l'incertitude commune en 
risque particulier, et qu'ils ont aussi transformé la participation collective au succès de la coopération en profit privatif. Ils ont donc «trahi » l'esprit de la coopération, qui était de constituer un acteur collectif, ils ont utilisé l'acteur collectif de manière privative.

Que les profiteurs soient de la première ou de la seconde espèce, la tendance, alors, est de supposer qu'ils forment un groupe adverse qui a procédé à un complot. Cela implique que ses membres étaient liés par des contraintes suffisamment fortes (des sanctions en cas de transgression) pour que leur coopération se réduise à une simple coordination, l'incertitude étant éliminée par le poids des sanctions. C'est une manière de dénier qu'en fait, les incertitudes étaient aussi grandes, voire plus, du côté de ces exploiteurs supposés.

Mais quelles qu'aient été les incertitudes assumées par les exploiteurs, et justement, paradoxalement, parce qu'elles pouvaient être plus grandes que celles assumées par les exploités, il reste qu'ils ont, par leur exploitation, aspiré en quelque sorte à leur profit l'incertitude agie, et qu'ils ont donc transformé l'incertitude agie de la coopération de ceux qu'ils exploitent en passivité partielle, puisqu'eux-mêmes ont su au contraire manipuler les risques. Ils ont fait de coopérateurs méritants des pigeons exploités, alors qu'ils tiraient les marrons du feu.

Le ressentiment des exploités est donc aggravé quand ils découvrent que leur coopération active est transformée par les exploiteurs en un suivisme plus passif. C'est aussi ce qui se passe quand des gens allaient à leur travail de manière routinière, et découvrent - généralement au moment des licenciements - que leurs efforts de meilleure productivité ont été exploités - pour donner une plus grande valeur à la firme. Ils découvrent alors d'un seul coup qu'en fait, leurs activités de routine étaient de la coopération, et de la coopération exploitée. Certes, ils se sont laissé engager, pas par pas, dans ces efforts, sans les comprendre comme une coopération méritante, mais à présent ils s'aperçoivent qu'ils ont été doublement abusés.

Le groupe qui se sent exploité a peut-être l'illusion du complot, mais il ne se trompe pas en pensant que sa coopération effective a été transformée en situation subie. Pour retrouver ses capacités de transmutations d'incertitudes en coopérations, il ne peut plus se lancer dans une incertitude supérieure (sauf à tenter une révolution). Il a été en effet dépassé dans cette gestion de l'incertitude par les exploiteurs. Il doit donc tenter de trouver une justification à la réussite des exploiteurs qui ne soit pas du type coopératif. Deux justifications sont disponibles: soit les exploiteurs ont bénéficié de hasards favorables, soit ils sont liés par les contraintes et les chantages réciproques qu'implique une mafia et ses complots. Dans une mafia, chacun agit pour le groupe, mais il subit aussi toute action d'un des membres. Les émotions agies y ont donc immédiatement leur correspondant en émotions subies.

\section{LES LEÇONS DE L'EXEMPLE ESPAGNOL}

Appliquons ces catégories à l'analyse de la réaction récente de l'opinion espagnole à l'attentat de la gare de Madrid le 11 mars 2003. Il ne s'agit pas à proprement parler de délibération, mais plutôt de la dénégation par le pouvoir espagnol des capacités de délibération d'une partie de sa population. Nous pourrions parler 
de délibération démocratique «en creux ». Notre interprétation est que l'opinion espagnole s'est sentie récusée dans sa capacité délibérative, qu'elle en a éprouvé du ressentiment, et qu'elle l'a manifesté lors des élections qui étaient en principe promises au pouvoir en place. La leçon pourrait en être qu'un pouvoir politique ne devrait pas négliger les «ressources délibératives» de sa population.

Dès avant cet attentat, l'opinion était majoritairement défavorable à une intervention en Irak. Une partie non négligeable des Espagnols avait marqué son indignation quand les justifications de cette intervention s'étaient révélées de la désinformation, et cette indignation avait été renforcée par la poursuite de cette intervention malgré une opinion majoritaire hostile. Mais cela n'avait pas suffi à donner une majorité d'opposition, en raison du nationalisme d'Aznar, de son rôle pour donner une image plus forte de l'Espagne sur la scène internationale, et de ses résultats économiques. Ce qui a déclenché un basculement politique et une majorité d'opposition, ce ne sont sans doute pas les conséquences directement négatives de cette politique (l'attentat), mais le fait qu'ait été poursuivie, même en face des conséquences négatives évidentes, une tentative de déni, qui pouvait ainsi potentialiser tous les réticences précédentes.

Cela pouvait les potentialiser, parce que ce qui pouvait être jugé comme le refus de laisser tomber un projet mobilisateur (un projet nationaliste: gloire de l'Espagne sur la scène internationale) quand il y avait simplement des risques, devient alors un refus d'assumer les conséquences des risques encourus. Une autre attitude, qui aurait assumé ces conséquences, et maintenu les valeurs «aznariennes », attitude qui aurait consisté à affirmer: "les islamistes veulent faire payer à l'Espagne son retour sur la scène internationale, mais ils ne nous feront pas plier» n'aurait sans doute pas eu les mêmes effets ${ }^{2}$.

Le basculement politique peut alors être compris de la manière suivante. L'opinion admet un engagement pour un projet collectif en incertitude. La première coopération demandée est de surmonter les régionalismes ou les séparatismes pour assurer l'unité de la nation espagnole. Mais Aznar demande une coopération supplémentaire, dans le but d'assurer le retour de l'Espagne sur la scène internationale. Et pour cela il demande aux Espagnols d'accepter l'intervention militaire aux côtés des USA en Irak. L'effort de coopération demandé consiste précisément à accepter d'avance les émotions négatives liées aux dangers de l'intervention. Mais cela implique que l'on prenne aussi en compte, dans le cas où cela tournerait mal, ce que seront les émotions de comparaison négatives. Or Aznar présente d'abord l'attentat comme venant de l'ETA.

La lutte contre l'ETA faisait partie de la coopération nécessaire pour surmonter les séparatismes. Mais les Basques se sont divisés entre ceux qui voulaient une autonomie politique relative (comme les Catalans) et les irréductibles partisans de la lutte terroriste. L'ETA est maintenant considérée comme le résidu d'un combat dont les objectifs les plus raisonnables sont en voie d'être atteints. Le combat contre l'ETA n'est donc plus l'affaire de la coopération des citoyens madrilènes, catalans et autres, c'est l'affaire de la police ou de l'armée. Le danger de l'ETA est

2 On dit qu'Aznar n'a pas pu adopter cette attitude parce que les explosifs ayant servi à l'attentat avaient été fournis par les services spéciaux espagnols qui infiltraient les cellules Al quaidistes! 
devenu un danger subi, et non plus l'enjeu d'une coopération motivante. Face à l'ETA, les Espagnols ne se sentent pas engagés dans une coopération risquée, qui donnerait un avantage novateur, comme celui d'une réputation internationale. Ils vivent plutôt l'ETA comme un mal indéracinable, subi. Paradoxalement, les seuls efforts de coopération dans ce domaine sont plutôt ceux des personnes qui soutiennent l'ETA (peut-être par anti-coopération, selon nos termes).

Ainsi, en attribuant d'abord l'attentat à l'ETA, Aznar dégradait et déqualifiait les risques qu'il avait pourtant demandés aux Espagnols d'assumer dans un effort de coopération. Il reniait cet effort même, et le transformait d'émotion de coopération agie en émotion négative subie. Il rompait donc la coopération des Espagnols avec lui, il agissait comme un exploiteur, qui veut bien tirer les fruits de la coopération quand elle marche, mais qui ne veut pas partager les coûts de la coopération quand elle ne marche plus.

Il aurait pourtant pu diriger le ressentiment des Espagnols contre Al Quaida. Cet attentat suscitait bien en effet un ressentiment, puisque le terrorisme d'Al Quaida utilisait l'effort des Espagnols pour gagner un statut de puissance qui a son mot à dire sur la scène internationale, et l'exploitait pour réaffirmer ses capacités à frapper tous ceux qui aident les USA. Les Espagnols se révélaient avoir travaillé pour Al Quaida en lui donnant un prétexte pour frapper une démocratie occidentale pro-américaine. Leur coopération était exploitée pour un tout autre motif. Ici aussi, d'ailleurs, le ressentiment espagnol n'aurait pas tenu compte des risques pris par les terroristes islamistes. Il faut d'ailleurs noter que les Espagnols se révélaient donner des occasions de réussite à une organisation qui constitue essentiellement un acteur anti-coopératif (Al Quaida n'existerait pas sans la politique des USA).

Mais ce ressentiment, Aznar l'a réorienté contre lui-même et son parti. Les Espagnols se sont alors tournés vers ceux pour qui refuser d'aller en Irak était une action, et non plus une absence de politique étrangère. C'était la meilleure manière de rationaliser leur situation émotionnelle. Aznar avait semblé vouloir transmuer une émotion négative agie en émotion subie, il avait donc joué le rôle de l'exploiteur. Le choix des Espagnols de se ranger avec le PS, qui avait décidé de refuser l'engagement en Irak, permettait toujours de tenir cette émotion négative pour une émotion subie, mais sans avoir à transformer l'émotion agie de la coopération en émotion subie. Il suffisait en effet de rompre la coopération avec Aznar pour que l'émotion subie soit de la responsabilité certes du terrorisme, mais aussi d'Aznar. Et il devenait au contraire possible de vivre le ressentiment comme une émotion agie (si l'on était celui qui refusait l'engagement en Irak). Au lieu d'avoir la double émotion d'une émotion négative devant l'attentat et de l'amertume de l'exploitation (de la transmutation de l'agi en subi, qui opère une dévalorisation), les Espagnols pouvaient alors affaiblir leur émotion négative en émotion subie par des circonstances contraires, tout en étant du côté des acteurs du refus, par leur ressentiment.

Cela nous enseigne que dans la relation démocratique, il ne faut jamais dénier aux citoyens, acteurs par définition du débat démocratique, et auxquels le politique demande sans cesse leur coopération, leur rôle d'acteurs. Cela revient à leur dénier leur capacité de délibération démocratique, puisqu'on leur demande leur effort de coopération tout en les traitant comme des êtres passifs quand on rencontre des difficultés. Cela est auto-contradictoire, puisque le politique, par 
essence, consiste à demander aux acteurs un effort de coopération qu'ils ne peuvent consentir qu'en tant qu'acteurs.

Inversement, il semble que la démocratie représentative, parce qu'elle permet aux votants de faire basculer leur vote d'un parti à l'autre, leur offre un dispositif qui permet de transformer les émotions négatives, liées aux conséquences des actions d'un parti, en émotions subies, sans pour autant qu'ils doivent se considérer comme des exploités, comme des acteurs dont on dénie le rôle actif, puisqu'il leur suffit de se ranger du côté de ceux qui refusaient la politique précédente, pour retrouver des émotions agies. En conséquence, la démocratie sanctionne tous les politiques qui n'affirment pas leurs valeurs même dans l'adversité, qui se présentent comme agissant quand cela va bien et comme subissant quand cela va mal. Mais cela a aussi un effet moins positif : dès que les valeurs ne sont plus affirmées dans une résistance à l'adversité, dès que la motivation collective est moins forte, la démocratie entre dans un processus qui peut mener une politique nationale à l'inefficacité, parce qu'il conduit à des basculements de vote à chaque échéance.

Ces considérations nous permettent de cerner un peu mieux les conditions d'une démocratie délibérative. Pour qu'il puisse y avoir démocratie délibérative, il faut non seulement que les personnes concernées puissent d'exprimer, connaître les informations des autres, et voir leur position prise en compte. Il faut aussi que l'effort de coopération qu'ils acceptent en confrontant leur opinion à celle des autres, en s'exposant publiquement aux critiques, effort qui est motivant seulement s'ils peuvent participer en tant qu'acteurs à la constitution de l'acteur collectif qu'est la délibération, ne conduise pas à une décision dont les justifications reposent sur l'hypothèse qu'ils vont continuer telle routine ou se résigner à ce qu'ils subissent. Il est évidemment nécessaire de s'interroger sur les procédures les plus adaptées aux délibérations, mais il faut d'abord s'assurer que les conditions contextuelles, avant et après les discussions, les débats publics, les conférences de consensus, sont bien en accord avec les exigences émotionnelles propres à la démocratie délibérative.

Une conception de la démocratie délibérative qui se réduirait à l'analyse des argumentations, en supposant que soient réunies les conditions d'une argumentation non fallacieuse et que les partenaires jouent le jeu de l'argumentation rationnelle, et qui négligerait les émotions, serait irréaliste et conduirait à de graves échecs. Ceux qui utilisent les émotions auront toujours le dessus, au moins temporairement, et la prise du pouvoir par les nazis a amplement montré les limites de l'argumentation réduite à elle-même et qui ne s'est pas donné les moyens de prendre en compte les émotions. Si la délibération démocratique, sous ses diverses formes (débat public, conférences de consensus, pannel de citoyens argumentant sur un projet, et bien entendu les débats des parlements et des cours constitutionnelles), ne tient pas compte des émotions et en particulier laisse grandir des ressentiments sans en mobiliser l'énergie pour des tâches coopératives, elle restera une utopie, et prendre cette utopie pour la réalité serait faire courir à la démocratie de grands dangers.

Université Aix-Marseille I et Centre d'Epistémologie et d'Ergologie comparative, CNRS, Paris 